\title{
Spanier stellen der spezifischen Immuntherapie ein gutes Zeugnis aus
}

Die spezifische Hyposensibilisierung ist eine wirksame, kausale Behandlung bestimmter Typ-1-Allergien. Nach wie vor limitiert aber die Angst vor schweren Nebenwirkungen die weitere Verbreitung dieser Behandlungsform. Eine multizentrische Untersuchung sollte die Sicherheit und Verträglichkeit nun auch für die Anwendung unter Alltagsbedingungen dokumentieren.

n 14 Allergiezentren in Spanien wurden insgesamt 488 Patienten mit einem Durchschnittsalter von 20,9 Jahren (5 bis 54 Jahre) mit handelsüblichen, biologisch standardisierten Allergenextrakten entsprechend den Leitlinien für die subkutane Immuntherapie der European Academy of Allergology and Clinical Immunology sowie den Dosierungsrichtlinien der einzelnen Hersteller behandelt. 152 Patienten (32\%) hatten eine Rhinitis ohne Asthma und 336 $(68 \%)$ litten unter leichtem oder mittelschwerem Asthma mit oder ohne Rhinitis. Eingesetzt wurden Pollen- oder Milbenextrakte.

Die Studie beendeten 423 Patienten $(86,7 \%)$. Von den insgesamt 17.526 Einzelinjektionen gab es bei 17.368 (99,1\%) keine unerwünschte Reaktion, in 105 Fällen (0,6\%) kam es zu lokalen Nebenwirkungen. Bei $18 \mathrm{~Pa}$ tienten $(3,7 \%)$ traten 53 systemische Reaktionen auf, das entsprach $0,3 \%$ aller Injektionen. Alle systemischen So- fortreaktionen waren leicht oder moderat und sprachen auf Gegenmaßnahmen wie die Gabe von Adrenalin, Antihistaminika oder Beta-Agonisten an. Ein anaphylaktischer Schock, le-

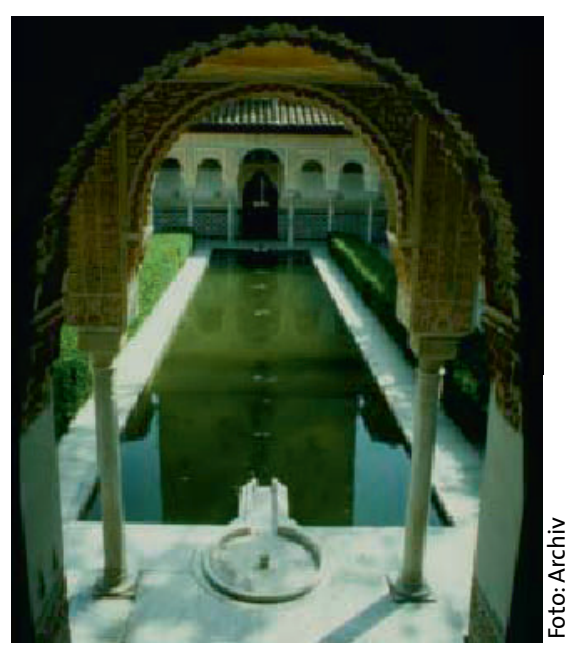

Eine neue multizentrische Studie gewährt Einblick in die in spanische Hyposensibilisierungspraxis. bensbedrohliche Reaktionen oder Todesfälle wurden nicht registriert.

Eine relativ erhöhte Nebenwirkungsrate ergab sich bei Patienten mit Asthma und bei der Verwendung von Milbenextrakten - ein aus anderen Studien bereits bekannter Befund. Ansonsten konnten keine neuen Risikofaktoren für das Auftreten von systemischen Reaktionen gefunden werden. Allerdings scheint es eine Subgruppe von Patienten zu geben, die - warum auch immer - besonders anfällig für Nebenwirkungen ist. Gehäufte Nebenwirkungen sind den Autoren zufolge als Warnhinweis einzustufen: Hätte man die Therapie nach Auftreten der dritten Nebenwirkung beim selben Patienten abgebrochen, wären in dieser Studie 21 Reaktionen (40\% aller registrierten systemischen Reaktionen) vermieden worden.

Fazit: Auch unter Praxisbedingungen ist - bei Einhaltung von Vorsichtsmaßnahmen und Beachtung von Kontraindikationen - die subkutane spezifische Hyposensibilisierung sicher. Eine noch größere Verträglichkeit bezüglich systemischer Reaktionen könnte erzielt werden, wenn bei gehäuftem Auftreten von Nebenwirkungen die Behandlung abgebrochen werden würde. $\quad b k$

Moreno C et al. Immunotherapy safety: a prospective multi-centric monitoring study of biologically standardized therapeutic vaccines for allergic diseases. Clin Exp Allergy 2004; 34: 527-31

\section{Sublinguale Hyposensibilisierung - hoher Plazeboeffekt bei Kindern}

\section{Die sublinguale Immuntherapie (SLIT) soll sich bei Kindern einer höheren Akzeptanz als die subkutane Variante erfreuen. Doch gerade für diese Patientengruppe ist die Wirksamkeit nicht ausreichend belegt - so auch das Ergebnis einer Cochrane-Analyse aus dem Jahr 2003. Nun gibt es aus Deutschland eine neue Multizenterstudie zur SLIT bei Kindern.}

llergologen und Pädiater der RuhrUniversität Bochum und der Universität Witten/Herdecke bezogen 161
Kinder mit einem Durchschnittsalter von 9 Jahren in eine insgesamt 3-jährige Untersuchung ein. Alle Probanden litten unter einer saisonalen Rhinokonjunktivitis und reagierten im Pricktest positiv auf Graspollen, 68 Kinder hatten zusätzlich Asthma. Die Studie wurde im 1. Jahr doppelblind, plazebokontrolliert und randomisiert durchgeführt, es folgte eine offene und kontrollierte Phase im 2. und 3. Jahr. Als Verum beziehungsweise Studienmedikation im 2. und 3. Jahr erhielten die Patienten sublingual Graspollenextrakte mit dem zehnfachen der Allergenmenge einer subkutanen Injektion.

Als primärer Endpunkt wurde in dieser Studie der klinische Index herangezogen, in dessen Berechnung sowohl 